\title{
Massenmedien als Diskriminierungs - und Propagandamittel am Beispiel von Jehovas Zeugen in der UdSSR
}

Ein wichtiger Bestandteil der Erforschung der Massenmedienpolitik in der ehemaligen UdSSR ist die Aufarbeitung der Geschichte von kleinen religiösen Minderheiten, die marginalisiert und verfolgt worden sind. Die historische Darstellung der Verfolgung von Jehovas Zeugen in Europa zeichnet gleichzeitig die Entwicklung der Religionsfreiheit in den demokratischen und totalitären Staaten, was den Kernpunkt des wissenschaftlichen Projekts „Jehovas Zeugen in Europa“ unter der wissenschaftlichen Betreuung von Gerhard Besier, des deutschen Historikers und ehemaligen Direktors des Hannah-Arendt-Instituts, bildet. ${ }^{1}$ Das Projekt begann 2007, und seine Ergebnisse wurden bisher in zwei enzyklopädischen Bänden unter dem Titel: „Jehovas Zeugen in Europa. Geschichte und Gegenwart“ veröffentlicht. ${ }^{2}$

* Doktorin der Geisteswissenschaften mit dem Schwerpunkt Literaturwissenschaft, promoviert an der Universität Lódź, Forschung auf dem Gebiet der Literaturwissenschaft, Kulturkunde und Geschichte, Teilnahme an internationalen Forschungsprojekten, unter anderem Mitarbeit am Projekt von Prof. Gerhard Besier ,Jehovas Zeugen in Europa. Geschichte und Gegenwart“. Lehrerin für Deutsch als Fremdsprache am österreichischen Bildungsinstitut (bfi) und Dolmetscherin sowie Übersetzerin für Polnisch und Deutsch in Österreich.

${ }^{1}$ Die andere Weltanschauung kann als die Verschiedenheit verstanden werden, die im Verhalten, Ideengut und in Glaubenssätzen zum Vorschein kommt und gesellschaftliche Abgrenzung zur Folge hat. Gruppen, die als ,anders” eingestuft werden, sind den weitgehenden Folgen von Intoleranz ausgesetzt, die - wie von der Forschung bestätigt - zur Einschränkung der Religionsfreiheit und zu großen Beeinträchtigungen für die Einzelnen führen, indem sie ihre Würde und fundamentale Menschenrechte verletzen. Zwar ist die religiöse Freiheit ein in allen demokratischen Staaten in der Verfassung verankertes Grundrecht, aber es gibt - so Gerhard Besier - in der praktischen Anwendung dieses Gesetzes große Differenzen. „Die Texte [der Veröffentlichung, J. H.] belegen, wie kleine Religions- und Weltanschauungsgemeinschaften ausgegrenzt, ihre Mitglieder beruflich benachteiligt und persönlich diffamiert werden." G. Besier, E. K. Scheuch (Hrsg.), Die neuen Inquisitoren. Religionsausübung und Glaubensneid, Band 1, Edition Interfrom/Fromm, Osnabrück, S. 25.

${ }^{2}$ Das sind: G. Besier, K. Stokłosa (Hrsg.), Jehovas Zeugen in Europa. Geschichte und Gegenwart, Band 1, LIT, Berlin 2013; G. Besier, K. Stokłosa, Jehovas Zeugen in Europa. Geschichte und Gegenwart, Band 2, LIT Berlin 2015. 
Der dritte Band, in dem unter anderem die Geschichte von Jehovas Zeugen in Polen abgehandelt wird, soll demnächst erscheinen.

Die Religionsfreiheit umfasst eine freie Meinungsäußerung, auch in den Medien. ${ }^{3}$ Um diese Freiheit mussten manche Gruppierungen ringen. Somit sind Ergebnisse der präsentierten Studie von Gerhard Besier - die historische Darstellung der Verfolgung und eines Kampfes einer relativ kleinen Religionsgemeinschaft um die Etablierung in der Gesellschaft, rechtliche Anerkennung und die Meinungsfreiheit - im Kontext der Medienpolitik äußerst interessant. ${ }^{4}$ Diese grundlegenden Menschenrechte litten besonders unter Diktaturen, in denen die Zensur kaum erlaubte, vom Staat aus als ,gefährlich“ oder „unerwünscht“ eingestufte Inhalte zu verbreiten. ${ }^{5}$

Durch die Analyse von Beeinflussungs- und Unterdrückungsmechanismen, deren sich Diktaturen durch Medien bedienten, wird die Vorgehensweise eines totalitären Staates bezüglich Andersdenkender erkennbar. Auch lassen sich Tendenzen zur Medienmanipulation und Einschränkung der freien Meinungsäußerung feststellen, die sich auch in demokratischen Ländern entwickeln könnten. ${ }^{6}$ Einem kurzen historischen Abriss folgt in dieser Studie die wissenschaftliche Analyse der gesammelten Dokumente. Zuletzt werden Schlussfolgerungen präsentiert.

${ }^{3}$ Die Meinungsfreiheit wird im Polnischen Grundgesetz in den Art. 14, 25, 49, 53 und 54 als Grundrecht festgelegt. Im Artikel 54 heißt es: „Jedem wird die Freiheit gewährleistet, seine Meinung zu äußern, Informationen zu holen und zu verbreiten. Zensur zu Präventionszwekken und Lizenzierung der Presse sind verboten." Konstytucja Rzeczypospolitej Polskiej (Polnisches Grundgesetz) vom 2. April 1997, S. 10. Quelle: http://isap.sejm.gov.pl/DetailsServlet?id=W DU19970780483, Zugriff vom 14.10.2016.

${ }^{4}$ In den postmodernen Gesellschaften zeichnet sich eine interessante Entwicklung ab, die man am Beispiel der Geschichte von Jehovas Zeugen, mitverfolgen kann. Die Forschungsergebnisse des Projektes unter der Betreuung von Gerhard Besier wurden - wie bereits erwähnt - in zwei Bänden veröffentlicht. (Vergleiche Fußnote Nr. 2). Bezüglich der Lage von kleineren Glaubensgemeinschaften in den demokratischen Ländern heißt es dort: „Auch wenn interessierte politische Kreise in Zusammenarbeit mit den etablierten Kirchen ,Diffamierungskampagnen“ und üble Nachrede im Hinblick auf kleine Religionsgemeinschaften betreiben, um die Gesellschaft gegen die ,Anderen` aufzuwiegeln, mit dem Ziel, das Feld allein zu beherrschen, werden solche Agitationen eine funktionierende Rechtsprechung - besonders auf höherer Ebene - letztendlich kaum beeinflussen. (...) Solche Verhältnisse sind in den Nordischen Ländern gegeben. (...) viele Länder - soweit sie in die Europäische Gemeinschaft aufgenommen wurden - sind auf dem Weg dorthin (...). In anderen europäischen Regionen - wie früher in der UdSSR, so nun in Russland und den GUS-Staaten häufen sich schwere Menschenrechtsverletzungen, namentlich solche, die die Religionsfreiheit betreffen.” G. Besier, K. Stokłosa, a.a.O., Bd. 2, S. 10.

${ }^{5}$ Zur Verletzung der Religionsfreiheit und des Rechtes auf freie Meinungsäußerung kommt es - so G. Besier und E. K. Scheuch - aufgrund der kommunikationspsychologischen Mechanismen. „Religiöse und sonstige Überzeugungen erhalten durch Meinungsführer von Interessengruppen stark negativ besetzte Etikettierungen (...). Diese beseitigen Hemmschwellen, rechtfertigen Kontrollmaßnahmen und befördern die Konsolidierung von Mehrheiten gegen Minderheiten." G. Besier, E. K. Scheuch, a.a.O., S. 25.

${ }^{6}$ Vgl. G. Besier, K. Stokłosa, a.a.O, Bd.2, S. 7f. 


\section{Die Geschichte von Jehovas Zeugen in der UdSSR im Überblick}

Die Aufarbeitung der Geschichte von Jehovas Zeugen in der UdSSR bleibt bis jetzt ein Desirat der Forschung. Der Zugang zu Archiven ist erschwert, und obwohl Zeitzeugen über ein gewisses Dokumentationsmaterial verfügen, besitzen sie keine schikanierenden Zeitungsartikel bzw. Aufzeichnungen von entsprechenden Radio- oder Fernsehsendungen.

Anfänge der Glaubensgemeinschaft gehen bereits auf die 1880er Jahre zurück, als der aus Lwiw stammende Ukrainer Semjon Koslicki in Zentralrussland als Bibelforscher aktiv war, was 1892 zu seiner Verbannung nach Sibirien führte. ${ }^{8}$ Am 20. Januar (2. Februar) 1918 wurde ein Dekret über die Freiheit des Gewissens, der Kirchen und religiösen Gemeinschaften herausgegeben. Es lautete u.a.: „Jeder Bürger darf sich zu irgendeiner Religion oder zu keiner Religion bekennen. Alle Gesetzesübertretungen, die mit dem Bekenntnis zu irgendeinem Glauben oder mit dem Bekenntnis zu keinem Glauben zu tun haben, werden aufgehoben." Allerdings verstärkte sich nach Lenins Tod der Kampf gegen die Religion. Im Jahre 1926 wurde der „,Verband der kämpfenden Gottlosen“ gegründet, um atheistische Propaganda zu verbreiten. Der Verband veröffentlichte antireligiöse Literatur wie die Zeitschrift „Antireligiosnik“ ${ }^{* 10}$

Mit Beginn des Zweiten Weltkrieges brach die regelmäßige Verbindung zu dem polnischen Büro in Lodz ab, von wo bis dahin die Tätigkeit von Jehovas Zeugen im Osteuropa koordiniert worden war. Die Zeugen wurden also gezwungen, ihr Religionswerk in den Untergrund zu verlegen. Bereits 1941 wurden mehrere Hundert Zeugen Jehovas aus Wolynien, Galizien, Bessarabien und Nordbukowina nach Zentralrussland, westlich und östlich des Urals, in Arbeitslager des Gulag, deportiert. ${ }^{11}$ Nach dem Zweiten Weltkrieg kamen nach Russland viele Zeugen Jehovas - russische Staatsbürger, die aus deutschen Konzentrationslagern befreit worden waren und dort mit dem Gedankengut der Zeugen Jehovas in Kontakt kamen und ihre Ansichten annahmen. ${ }^{12}$

\footnotetext{
${ }^{7}$ Jahrbuch der Zeugen Jehovas 2008, herausgegeben von Wachtturm - Bibel- und Traktatgesellschaft, Selters 2008, S. 67f.

${ }^{8}$ Es war nicht ein einziger Verfolgungsakt aufgrund der Religionsausübung. Vgl. die Aussage von Roman Sitartszuk in: W. Slupina, K. Berezhko, Die Diktaturerfahrung von Jehovas Zeugen in der Ukraine als Teil ihrer Verfolgungsgeschichte in der UdSSR, in: G. Besier, K. Stokłosa, a.a.O., Bd. 2, S. 540.

${ }^{9}$ Ebd., S. 20.

${ }^{10}$ Dort konnte man 1928 lesen: „Oblast Woronesch wimmelt von Sekten.” Erwähnt wurden unter anderem 48 ,Erforscher der Heiligen Schrift“ und deren „Leiter Sintschenko und Mitrofan Bowin“. Jahrbuch, a.a.O., S. 81.

${ }^{11}$ Vgl. K. Biliński, Hiobowie XX wieku. Wspomnienia Świadków Jehowy (Badaczy Pisma Świętego), A PROPOS, Wrocław 1998, S. $102 \mathrm{ff}$.

12 Die KZ-Häftlinge aus Russland und der Ukraine kamen im Lager nach einem langen Transport entkräftet und erschöpft an. Ihr Überleben hing von der solidarischen Hilfe der anderen
} 
Im Jahr 1945 gab es auf dem Gebiet der Sowjetunion über 1600 Zeugen Jehovas; ein Jahr später (1946) stieg ihre Anzahl auf 4797. ${ }^{13}$ Zum damaligen Zeitpunkt unternahmen sie einige Bemühungen um die offizielle Registrierung der Glaubensgemeinschaft, die jedoch erfolglos blieben. In Antwort auf den Antrag, gestellt beim Präsidium des Obersten Sowjets der UdSSR in Moskau hieß es:

Alle Zeugen Jehovas, die bereits sind, mit den Staatsorganen zusammenzuarbeiten, sollen freigelassen werden; Jehovas Zeugen dürfen ihre Literatur legal (aber nach der Zensur der Staatsorgane) in Druckereien der Sowjetunion drucken, sie dürfen legal die Bibel benutzen, aber nur innerhalb des eigenen Wohnsitzes (der Wohnung); die Verbindung mit dem Hauptbüro der Zeugen Jehovas in Brooklyn sollte erlaubt werden, aber das darf nur durch die offiziellen Kanäle der UdSSR geschehen. ${ }^{14}$

Außerdem verlangte die Regierung die Herausgabe aller Namen und Adressen der Zeugen Jehovas in der UdSSR und stellte darüber hinaus die Bedingung, dass „Jehovas Zeugen in der Sowjetunion die existierende Ideologie als richtig akzeptieren und den Aufbau der kommunistischen Gemeinschaft propagieren“. ${ }^{15}$ Da dies den Glaubensprinzipien von Jehovas Zeugen widersprochen hätte, konnten sie den Bedingungen nicht zustimmen, was dazu führte, dass sie von der sowjetischen Regierung als „Feinde“ betrachtet und „zu den ,antistaatlichen, antisowjetischen und fanatischen Sekten' gerechnet und nicht als Religionsgemeinschaft, sondern als politische Gruppe eingestuft ${ }^{\star 16}$ wurden. Aus dem Grund

Häftlingen ab. Die Historikerin Kirsten John-Stucke berichtet, dass russische Häftlinge vor allem von den Bibelforschern und politischen Häftlingen Hilfe bekamen. Sie wurden vor allem zu Baracken von Bibelforschern verlegt, die sogar im Lager ihr Missionierungswerk fortführten. Um die Sprachbarrieren zu überwinden gebrauchten sie sogenannten „Zeugniskarten”, die in verschiedene Sprachen übersetzt worden sind. Es gelang ihnen sogar, biblische Vorträge abzuhalten. Im Endeffekt wurden viele Häftlinge aus Russland und der Ukraine selbst Zeugen Jehovas. Vgl. W. Slupina, K. Berezhko, a.a.O., Bd. 2, S. 556.

${ }^{13}$ Vgl. ebd., S. 559.

${ }^{14}$ Ebd., S. 566.

15 Ebd.

${ }^{16}$ Ebd, S. 560. Die Vorgehensweise, einer Religionsgemeinschaft politischen Charakter zu unterstellen, schaffte einen Grund für die Repressionen von Seiten der Sowjetregierung. Mit der Methode ging man gegen Jehovas Zeugen in den verschiedenen kommunistischen Ländern vor. Bis zum Jahre 1950 verbot man ihre Tätigkeit in der DDR, Tschechoslowakei, in Polen, Albanien, Rumänien, Bulgarien und Ungarn. In der kommunistischen DDR kam es zu landesweiten Kampagnen und Restriktionsmaßnahmen. Die Hauptanklage lautete: Tätigkeit mit umstürzlerischem Charakter. So stellte der stellvertretende Ministerpräsident Otto Nuschke in einem Referat zur politischen Lage in der DDR fest, dass es sich bei den Zeugen Jehovas ,um keine religiöse Bewegung, ja nicht einmal um eine Sekte handelt, sondern um eine sich lediglich religiös tarnende politische Untergrundbewegung“. Tägliche Rundschau vom 07.09.1950, Nr. 209, zitiert nach: G. Yonan, Jehovas Zeugen. Opfer unter zwei deutschen Diktaturen 1933-1945; 1949-1989, Konkordia Druck, Berlin 1999, S. 84ff. 
standen sie ,ständig unter Observierung durch staatliche Sicherheitsorgane und man versuchte, ihre Druckereien und Literaturdepos im Untergrund ausfindig zu machen sowie die Verbindungswege ins Ausland aufzuspüren." ${ }^{\text {"17 }}$

An der Stelle soll bemerkt werden, dass die Sowjetregierung nicht vorhatte, die Zeugen auszurotten, sondern sie vielmehr für die Sowjetideologie zu gewinnen - entweder durch Überzeugungsarbeit oder mit Gewalt. Dazu wurde der Geheimdienst - der KGB - eingesetzt, der sich unterschiedlicher Methoden bediente. $\mathrm{Zu}$ denen gehörten: Durchsuchungen ${ }^{18}$, Bespitzelungen ${ }^{19}$, Geldstrafen und Auflösung der Zusammenkünfte ${ }^{20}$, Bestechung und Erpressung ${ }^{21}$, Beschlagnahmung von Besitz sowie Familientrennung ${ }^{22}$, Spott, Hiebe ${ }^{23}$, Inhaftierung und Einweisung in Arbeitslager ${ }^{24}$. Um die Strukturen der Religionsgemeinschaft von innen aus zunichte zu machen und ihre Einheit zu zerstören, arbeitete der Sicherheitsdienst eine Strategie „der inneren Zersetzung“ aus. Die Vorgehensweise war, die KGB-Agenten in die Reihen der Zeugen einzuschleusen, um wichtige Informationen weiterzugeben und somit Misstrauen und Zwietracht unter den Zeugen zu säen. ${ }^{25}$

${ }^{17}$ W. Slupina, K. Berezhko, a.a.O., Bd. 2, S. 560.

${ }^{18}$ Durchsuchungen wurden tagtäglich durchgeführt. Vgl. O Golko, Świadkowie Jehowy. Sybirski trakt, A PROPOS, Wrocław 2004, S. 143.

${ }^{19}$ In den Wohnungen von Zeugen wurden heimlich Abhörgeräte eingebaut. Vgl. Jahrbuch, a.a.O., S. 133 .

${ }^{20}$ Immer wieder fand man heraus, wo die Zusammenkünfte stattfanden. Alle Anwesenden bekamen Geldstrafen, die oftmals mindestens die Hälfte eines durchschnittlichen Monatslohns ausmachten. Ebd., S. 92.

${ }^{21}$ Vergleiche die Zeitzeugenaussagen in der Videodokumentation: „Treu angesichts der Prüfungen. Jehovas Zeugen in der Sowjetunion“, herausgegeben von Watch Tower and Tract Society of Pennsylvania, Brooklyn (New York), 2003/2008.

${ }^{22}$ Vgl. O. Golko, a.a.O., S. 208f. Vgl. auch W. Slupina, K. Berezhko, a.a.O., S. 663. Die Kinder von Jehovas Zeugen sollten im Sinne der kommunistischen Ideologie zu aktiven Kommunisten „umerzogen“" werden. Vgl. Ebd.

${ }^{23}$ Vgl. Jahrbuch, a.a.O., S. $114 f$.

${ }^{24}$ W. Slupina, K. Berezhko, a.a.O., S. $575 f f$.

${ }^{25}$ Bespitzelung war eine Maßnahme zur Zersetzung der Organisation und Zerstörung ihrer Strukturen. Gabriele Yonan stellte fest: „Im Rahmen der Aktion ,Zerfall` sollte auf der psychologischen Ebene Unsicherheit und Zersplitterung in die Religionsgemeinschaft der Zeugen in der DDR getragen werden“. G. Yonan, Im Visier der Stasi. Jehovas Zeugen in der DDR, edition corona, Niedersteinbach 2000, S. 19. Über die Aktion selbst heißt es in den Geheimakten der DDR: „Die Aktion ,Zerfall' muss so geführt werden, dass die Mitglieder der , ZJ`' ständig in dem Glauben sind, dass jede Handlung, die sie für die Sekte tun, von den Sicherheitsorganen überwacht wird. Dabei kommt es besonders darauf an, die Meinung aufkommen zu lassen, dass gerade Personen aus den Spitzen der Sekte die Sicherheitsorgane informieren. Die Angehörigen dieser Sekte muss man spüren lassen, dass ihre sogenannte Verkündigertätigkeit, die Durchführung von Hausbesuchen und überhaupt die gesamte illegale Tätigkeit für die ,ZJ' mit großen Gefahren verbunden ist, ja sogar zur Festnahme führen kann.“ BstU MfS BV Gera 857/80, Bd. 1, S. 247ff. Quelle: G. Yonan, Im Visier, a.a.O., S. 19. Die KGB-Agenten begannen, die Bibel zu studieren, sich an 
Die Medien waren ein mächtiges Instrument zur Verbreitung des Kommunismus und des atheistischen Gedankengutes. Karin Moser macht in ihrer Studie darauf aufmerksam, dass die Sowjetunion bereits nach dem Ersten Weltkrieg einen mächtigen Kontrollapparat entwickelte, der aus Zensur und gelenkter Entwicklung der Massenmedienpropaganda bestand. Dabei stellt Karin Moser fest:

Die ursprüngliche, aus dem Lateinischen stammende, Bedeutung des Terminus „Propaganda“ - ausbreiten, verbreiten und erweitern - wurde in der politischen Praxis erweitert. Die noch relativ neutrale Wertung, wonach man unter Propaganda die Übertragung von Ideen oder Werten von einer Person oder einer Gruppe von Personen zu anderen versteht, erfuhr in Verbindung mit dem Begriff „Manipulation“ eine pejorative Bedeutungsverschiebung. Manipulation weist hierbei auf die missbräuchliche Nutzung der meinungsbildenden Wirkung der Massenmedien zur einseitigen Beeinflussung der Konsumenten. ${ }^{26}$

Die Deutung dieser Termini ist sowohl politisch als auch gesellschaftlich motiviert. Politische Propaganda wird dagegen als ,gezielte Handlung“ bezeichnet, mit dem Ziel, ,das politische Verhalten der Einzelnen im Interesse der Herrschenden zu beeinflussen“"27.

Jehovas Zeugen wurden in der sowjetischen medialen Darstellung als Feinde präsentiert. Zu bemerken ist, dass die bildliche Darstellung eines Feindes sowie die bildliche und sprachliche Teilung der Welt in Freund und Feind zu den wichtigsten Themen der Massenmedien gehörten. Das Konstrukt des Feindes wurde unter anderem auch durch Vorträge in Gefängnissen und Lagern propagiert, die vermittelten, Jehovas Zeugen würden unter dem religiösen Mantel eine umstürz-

der Predigttätigkeit zu beteiligen und Voraussetzungen, die den Angehörigen der Glaubensgemeinschaft gestellt werden, zu erfüllen. In der DDR und in Polen wandte man ähnliche Methoden an. Die Spitzel hatten die Aufgabe, Behörden über die Tätigkeit einzelner Gruppen und deren Zusammenkünfte zu informieren sowie Namen der Zeugen weiterzugeben. Vgl. auch: W. Slupina, Prześladowania i represje Świadków Jehowy w Polsce w latach 1939-1945 oraz 1950-1989 in: H. Hesse (Hrsg.), „Najodważniejsi byli zawsze Świadkowie Jehowy...” Prześladowania i sprzeciw Świadków Jehowy w okresie reżimu hitlerowskiego, A PROPOS, Wrocław 2006, S. 226ff. Vgl. auch: W. Hirsch (Hrsg.), Zersetzung einer Religionsgemeinschaft. Die geheimdienstliche Bearbeitung der Zeugen Jehovas in der DDR und in Polen, edition corona, Niedersteinbach 2001.

${ }^{26}$ K. Moser, Propaganda und Gegenpropaganda-das kalte Wechselspiel während der alliierten Besatzung in Österreich, in: medien \& zeit, Nr. 1/2002, S. 27 oder unter: www.demokratiezentrum.org. Die Deutung dieser Termini ist sowohl politisch als auch gesellschaftlich motiviert. In der Sowjetunion waren deshalb die Begriffe „Propaganda” und „Agitation” positiv gewertet, weil man durch die Verbreitung der ,einzig gültigen Wahrheit“ einen Beitrag zum ,Allgemeinwohl“ geleistet zu haben sah, indem man den sozialen und gesellschaftlichen Wandel vorantrieb. Vgl. ebd.

${ }^{27}$ L. Wojtasik, Psychologia propagandy politycznej, Warszawa 198, S. 32-34; zitiert nach: A. Wirowska, Animowane filmy jako narzędzie propagandy politycznej w ZSRR w latach 1941-1942, in: Doctrina. Political and Scientific Studies 7/2010, s. 197-206. Die Datei ist abrufbar unter: www. academia.edu 
lerische Tätigkeit durchführen. ${ }^{28}$ Diese mediale Botschaft verstärkten auch Fil$\mathrm{me}^{29}$ und Gerichtsprozesse, über die im Fernsehen und in der sowjetischen Presse ausführlich berichtet wurde.

Im Jahr 1960 spielte Wenera Grigorjewa die Hauptrolle in einem sogenannten „Dokumentationsfilm“ „Gottes Zeugen“, der in Kinos in der Sowjetunion gezeigt wurde..$^{30}$ Die Religionsgemeinschaft wurde als „,furchtbar“ und ,erschreckend" dargestellt und wird des Todes der Hauptheldin beschuldigt. Tanja Wiesielowa flieht in der Nacht von den Sektenangehörigen und verschwindet in einem Schneesturm. ${ }^{31}$ Die Schauspielerin selbst betont, wie stark solche Filme die Meinung der Zuschauer beeinflussten:

Der Film wurde in den Kinos und Klubs vieler Städte gezeigt. Ich war bei jeder Premiere dabei und zeigte mich danach auf der Bühne. Damals nahmen die Menschen in der Sowjetunion alles, was ihnen im Fernsehen oder Kino gezeigt wurde, für bare Münze. Wenn ich also auf die Bühne kam, stießen viele einen Erleichterungsseufzer aus und riefen: „Sie lebt!“ Ich erklärte ihnen dann, wie wir den Film gedreht hatten und wie der Regisseur Spezialeffekte eingesetzt hatte - beispielsweise den Schneesturm, in dem ich angeblich in eine Schlucht stürzte und unter dem Schnee begraben wurde. ${ }^{32}$

In der Sowjetunion hatte man etliche Filme gedreht, die die Glaubensgemeinschaft diskreditieren sollten, unter anderem: „Harmagedon“, „Beichte“, „Ein Sünder“, „Gottes Zeugen“, „Apostel demaskiert“.33 Im Lebensbericht vom Zeitzeugen L. Toom wurde auch eine satirische Komödie erwähnt, aufgeführt am Estnischen Theater in Tallin. ${ }^{34}$

${ }^{28}$ Den Vorwurf, unter dem religiösen Mantel politisch aktiv zu sein, brachte man gegen Jehovas Zeugen auch in NS-Deutschland vor. Vgl. Anmerkung Nr. 16.

${ }^{29}$ Die Handlung des Filmes „Harmagedon” (1962) sollte die Assozialität der Zeugen Jehovas samt ihrer antagonistischen Haltung herausstellen sowie auch die „Wahrheit” über diese Glaubensgemeinschaft und geheimnisvollen sowie politischen Charakter enthüllen.

${ }^{30}$ W. Slupina, K. Berezhko, a.a.O., Bd.2, S. 652. Werena Girgojewa erzählt: „Zu Beginn meiner schauspielerischen Karriere im Jahr 1960 erhielt ich eine Hauptrolle in einem Dokumentarfilm, der übersetzt „Gottes Zeugen” hieß und in den sowjetischen Kinos lief. Der Film drehte sich um die ,schauerliche Sekte der Zeugen Jehovas', die für den Tod der von mir gespielten Filmheldin Tanja verantwortlich war. Laut Drehbuch läuft Tanja mitten in der Nacht in einem Schneesturm ohne Mantel vor der ,Sekte davon. Während sie im Schnee verschwindet, sagt der Kommentator mit Grabesstimme: ,Das war das Ende von Tanja Wiesielowa‘. Mir gefiel das Drehbuch und es war mir eine Ehre, im Kampf gegen die Zeugen mit von der Partie zu sein, auch wenn ich sie nur vom Drehbuch her kannte.“ Das Jahrbuch, a.a.O., S. 214.

${ }^{31}$ Ebd, S. 215.

${ }^{32} \mathrm{Ebd}$.

${ }^{33}$ W. Slupina, K. Berezhko, a.a.O., S. 652.

${ }^{34}$ Ebd. 
Am Beispiel des Filmes „Harmagedon“ wird die Methodologie der Medienpropaganda sichtbar. Sie basiert auf eindimensionaler Zeichnung von Charakteren, Ungenauigkeit und Halbwahrheiten. ${ }^{35}$ Die Hauptrolle spielt Mutter von zwei Kindern, die eine Zeugin und - obwohl gläubig - kühl, distanziert, hinterlistig, verrückt, gewalttätig und feindselig ist. Nur in wenigen Momenten kommen ihre natürlichen mütterlichen Gefühle zum Ausdruck, und zwar als sie vom Tod ihrer kleinen Tochter erfährt, die jedoch in religiösen Wahnsinn umschlagen. Religionsausübung wird als mystischer Wahnsinn dargestellt, verstärkt durch Musik, Lichteffekte und Darstellung. ${ }^{36}$ Sowjetische Propagandafilme sollte man vor dem ideologischen Hintergrund ihrer Entstehungszeit betrachten. Anna Wirowska bemerkt, dass

die Botschaft für den Empfänger klar und deutlich sein musste. Das Ziel der Propaganda ist, das Leben des Einzelnen so weit wie möglich zu beeinflussen. [...] Durch die propagandistischen Maßnahmen wird die Veränderung des menschlichen Verhaltens angestrebt, und zwar so weit, wie das vom Sender der Botschaft - hier also von der Sowjetischen Kommunistischen Partei - bestimmt wird. ${ }^{37}$

Als ein mächtiges Massenmedium in der Sowjetunion gilt auch die Presse. Bereits Lenin forderte die Nutzung von Zeitungen als „Organe der verschiedenen Parteiorganisationen“ und Kontrolle ihrer Inhalte. ${ }^{38}$ Sozialistischer Journalismus hatte damit die „Richtlinien der Partei“ zu befolgen. „Jeder sollte die Darstellungen der sozialistischen Gesellschaft verstehen, deswegen wurden Vereinfachungen und Verdeutlichungen im sozialistischen Realismus bevorzugt ${ }^{\star 39}$ - heißt es über die Literatur der damaligen Zeit. In der Literatur wurde dafür ein positiver Held benötigt, der aus dem Arbeitermilieu kam oder Verbindung zu ihm hatte. Wichtig war seine optimistische Grundhaltung, die mit dem Weltbild des Kommunismus übereinstimmen sollte. „Experimente, Mystisches und Themen aus dem religiösen Bereich sollten im sozialistischen Realismus vermieden werden. ${ }^{40}$

${ }^{35}$ In der ukrainischen Zeitung „Tscherwonyj prapor” vom 14. Mai 1963 erschien eine Filmkritik, in der es hieß: „Auf solche Weise ist die atheistische Propaganda wirksam, überzeugend, und sie kann in anderen Ortschaften des Landes gebraucht werden, wo ähnliche Filme gezeigt werden." Zitiert in: Slupina, K. Berezhko, a.a.O., S. 652.

${ }^{36}$ Der Film ermöglicht, Emotionen zu beeinflussen. A. Wirowska kommentiert dies wie folgt: „Der visuelle Transfer übertrifft andere Propagandaformen insofern, dass man durch Bilder und Ton den Schwerpunkt auf die emotionelle Botschaft und nicht so sehr auf Informationen setzen kann. Ein zusätzlicher Vorteil der Filmproduktion ist, dass sie in der gleichen Zeit viel mehr Informationen liefern kann. In der Folge kann der Zuschauer - trotz der Ablehnung von Inhalten - durch Beeinflussung des Unterbewusstseins - diese auf der emotionellen Ebene annehmen. A. Wirowska, a.a.O, S. 198.

${ }^{37}$ A. Wirowska, a.a.O., S. 198.

${ }^{38}$ H. Budzislawski, Sozialistische Journalistik. Eine wissenschaftliche Einführung, Leipzig 1966, S. $103 f$.

${ }^{39}$ Ebd.

${ }^{40}$ Ebd. 
Von der Staatssicherheit in der UdSSR wurde Ende 1975 eine Desinformationskampagne eingeleitet. Dazu heißt es in einem geheimen Informationsschreiben vom 20. August 1976:

Wesentliches Augenmerk [sei] auch auf die Vorbeugungs- und Aufklärungsarbeit unter den Sektenmitgliedern mit Hilfe der Öffentlichkeit und der Masseninformationsmittel gerichtet [...]. Im Einzelnen wurden in der periodisch erscheinenden Presse ca. 250 Artikel veröffentlicht, über 100 Radio- und Fernsehsendungen organisiert und überall mit Sektenanhängern thematische Abende, Diskussionen, Treffen in Dörfern und öffentlichen Versammlungen veranstaltet. ${ }^{41}$

Berichte über Jehovas Zeugen, die in der regionalen Presse erschienen, können in zwei Kategorien unterteilt werden. Zur ersten Kategorie gehören Berichte, die stark politisiert waren oder direkt im Auftrag von staatlichen Stellen verfasst wurden. In die zweite Kategorie fallen Informationen, die der atheistischen Propaganda dienen sollten oder „Entlarvungscharakter“ hatten. Die Berichterstatter dieser Kategorie zeichneten die Zeugen Jehovas in den "schwärzesten Farben“ und säten Misstrauen in der Gesellschaft. In der Presse wurde umfangreich von Gerichtsprozessen berichtet. ${ }^{42}$ Der Gerichtssaal wurde hiermit zum Medium, durch das die Öffentlichkeit (des)informiert werden sollte. In der Sowjetunion gab es zwei Arten von Prozessen: die Verhandlung vor dem Gericht und das sogenannte „Towarischetschski sud“ - das Genossengericht ${ }^{43}$, bei dem es sich um eine Einrichtung handelte, die Bestandteil des sowjetwischen Systems zur Erziehung der Bürger war. ${ }^{44}$ „Parteifunktionäre sahen in diesen ,Genossengerichten eine gute Möglichkeit, Zeugen Jehovas wegen ihrer Religion zu diffamieren und gleichzeitig das Sowjetsystem und die kommunistische Partei propagandistisch zu verherrlichen. ${ }^{\star 45}$ Konstantin Berezhko resümiert wie folgt:

Die Berichterstattung der sowjetischen Presse bediente sich [...] folgender Elemente: Falschdarstellungen (das betraf unter anderem die Glaubenslehren); unrealistische Einschätzungen der Situation im Land (z. B. die marxistisch-leninistische Theorie

${ }^{41}$ W. Slupina, K. Berezhko, a.a.O., S. 669.

42 Ebd.

${ }^{43}$ Ebd., S. 653.

44 „Gewöhnlich fand das Prozedere in einem Gemeindesaal statt oder, falls der Angeklagte ein Fabrikarbeiter war, z.B. im Speisesaal der Fabrik. Dort stand auf einer Bühne ein großer Tisch mit rotem Tischtuch, an dem die Ankläger saßen. Auf dem Tisch ,thronte' oft eine Leninbüste und dahinter hingen Spruchbänder und Plakate, die den Sozialismus priesen. Die ersten Reihen des Auditoriums waren besetzt von Parteifunktionären sowie Vertretern der örtlichen Presse und des Fernsehens. Denn anschließend wurde der Fall medienwirksam in der Presse ausgebreitet." Ebd.

${ }^{45}$ Ebd. 
vom Niedergang der Religion durch den Sozialismus); negative Bruchstücke einer Biografie, Apostatenberichte; Verallgemeinerung von Ausnahmen und schwer überprüfbare Behauptungen. ${ }^{46}$

\section{Bibliografia}

Berezhko K., Jehovah's Witnesses an the Mass Media in the Post-Soviet Period, [w:] On religious liberty in a democratic society. Aspects of law, religion and philosophy in constitutional theory and reality, "Religion - Staat - Gesellschaft" 2009, nr 10/2, s. 169-179.

Besier G., Stokłosa K. (red.), Jehovas Zeugen in Europa. Geschichte und Gegenwart, t. 1, Wydawnictwo LIT, Berlin 2013.

Besier G., Stokłosa K. (red.), Jehovas Zeugen in Europa. Geschichte und Gegenwart, t. 2, Wydawnictwo LIT, Berlin 2015.

Biliński K., Hiobowie XX wieku. Wspomnienia Świadków Jehowy (Badaczy Pisma Świętego), Wydawnictwo A PROPOS, Wrocław 1998.

Budzisławski Hermann, Sozialistische Journalistik. Eine wissenschaftliche Einführung, Wydawnictwo Bibliographisches Institut Leipzig, Lipsk 1966.

Golko O., Świadkowie Jehowy. Sybirski trakt, Wydawnictwo A PROPOS, Wrocław 2004.

Hirsch W. (red.), Zersetzung einer Religionsgemeinschaft. Die geheimdienstliche Bearbeitung der Zeugen Jehovas in der DDR und in Polen, Wydawnictwo Edition Corona, Niedersteinbach 2001.

Hirsch W. Zusammenarbeit zwischen dem ostdeutschen und dem polnischen Geheimdienst zum Zweck der ,Zersetzung” der Zeugen Jehovas, [w]: Zersetzung einer Religionsgemeinschaft. Die geheimdienstliche Bearbeitung der Zeugen Jehovas in der DDR und in Polen, Wydawnictwo Edition Corona, Niedersteinbach 2001, s. 84-95.

Moser Karin, Propaganda und Gegenpropaganda - das kalte Wechselspiel während der alliierten Besatzung in Österreich, „Medien \& Zeit 2002, nr 1 (lub online na stronie: www.demokratiezentrum.org).

Scheuch Erwin K. (red.), Die neuen Inquisitoren. Religionsausübung und Glaubensneid, t. 1, Wydawnictwo A. FROMM, Osnabrück 1999.

Slupina Wolfram, Berezhko Konstantin, Die Diktaturerfahrung von Jehovas Zeugen in der Ukraine als Teil ihrer Verfolgungsgeschichte in der UdSSR, w: Besier G., Stokłosa K. (red.), Jehovas Zeugen in Europa. Geschichte und Gegenwart, t. 2, Wydawnictwo LIT, Berlin 2015, s. 533 - 690.

Slupina Wolfram, Jehovas Zeugen in der Gemeinschaft unabhängiger Staaten und in Georgieneine Bestandsaufnahme in der postsowjetischen Ära, w: Besier G., Stokłosa K. (red.), Jehovas Zeugen in Europa. Geschichte und Gegenwart, t. 2, Wydawnictwo LIT, Berlin 2015, s. 253-353.

Slupina Wolfram, Prześladowania i represje Świadków Jehowy w Polsce w latach 1939-1945 oraz 1950-1989, w: Hesse H. (red.), „Najodważniejsi byli zawsze Świadkowie Jehowy...” Prześladowania i sprzeciw Świadków Jehowy w okresie reżimu hitlerowskiego, Wydawnictwo A PROPOS, Wrocław 2006, s. 207-245.

Strażnica - Towarzystwo Biblijne i Traktatowe. Zarejestrowany Zwiazek Wyznania Świadków Jehowy w Polsce, „Rocznik Świadków Jehowy na rok 2008”, Selters 2008.

Watch Tower and Tract Society of Pensylvania (wyd.), Wierni w obliczu prób. Świadkowie Jehowy w Zwiąku Radzieckim (DVD), Brooklyn (New York), 2003/2008.

${ }^{46}$ Vgl. K. Berezhko, Jehovah's Witnesses an the Mass Media in the Post-Soviet Period, in: RSG 10/2 (2009), S. 169ff. Zitiert nach: W. Slupina, K. Berezhko, a.a.O., S. 670. 
Wirowska A., Animowane filmy jako narzędzie propagandy politycznej w ZSRR w latach 1941-1942, „Doctrina. Political and Scientific Studies” 2010, nr 7, s. 197-206.

Yonan G., Im Visier der Stasi. Jehovas Zeugen in der DDR, Wydawnictwo Edition Corona, Niedersteinbach 2000.

Yonan G., Jehovas Zeugen. Opfer unter zwei deutschen Dikaturen 1933-1945; 1949-1989, Wydawnictwo Konkordia Druck, Berlin 1999.

\section{Strony internetowe}

http://isap.sejm.gov.pl/DetailsServlet?id=WDU19970780483, dostęp z dnia 14.10.2016

http://www.jw.org/wiadomo\%C5\%9Bci/sytuacja-prawna/regiony/rosja/odwolanie-odrzucone-20161014/ Dostęp z dn. 24.10.2016

http://www.jw.org/pl/wiadomo\%C5\%Bci/sytuacja-prawna/regiony/rosja/delegalizacja-serwisu-jw-org/ Dostęp z dn. 24.10.2016

Justyna Haas

\section{Mass media as a means of discrimination and propaganda - based on the example of Jehovah's Witnesses in the USSR}

\section{(Summary)}

Jehovah's Witnesses were banned as a religious community in the USSR. It was only after the collapse of the Soviet Union that the first registrations of the recognized religious communities followed in the territory of the Soviet Republics. In the Soviet Union, the government used media as a propaganda tool to create the image of Jehovah's Witnesses as an anti-social and harmful group. They were accused of political activities, espionage to the US, and antisocial behavior. The films and press were used. The manipulation of information was based on agitations, false information, halftruths, political agitation and slander. The consequence of this was a wide-ranging discrimination against the witnesses. Their children got poorer grades, and employers refused to accept the money and leave. In order to win Jehovah's Witnesses for Communist ideology and to break down organizational structures, the security services of the Eastern Bloc were working together, and in particular the Ministry of State Security of the GDR made a great contribution to this area. After 1990, the persecuted Jehovah's Witnesses were rehabilitated. They received victim cards and compensation money.

Both in Russia and other postsovskaya republics there are tendencies to the progressive restriction of freedom of expression and religion. Since March 2015, the importation of literature from Jehovah's Witnesses to Russia has been banned. In July 2015, their official website was forbidden. The public prosecutor's office investigates some of Jehovah's Witnesses because of their religious activities. On July 17, 2017 the Supreme Court of Russia confirmed its earlier ruling that criminalized the activity of Jehovah's Witnesses in Russia. Jehovah's Witnesses have submitted 28 applications to the European Court of Human Rights (ECHR) because of the allegations of extremism and related maladministration. In 2015, the US Human Rights Commission reiterated its recommendations to Russia, which had previously been addressed (in 2003 and 2009), „to revise the law to combat extremist activities".

Keywords: discrimination, propaganda, Jehova's Witnesses, religion, politics, totalitarism. 